\title{
STUDY OF APPLICATION AND THE USES OF THE UNMANNED AERIAL VEHICLE USING INSECTICIDE SPRAYING
}

\author{
Santhosh .R ${ }^{1}$, Senthil. $\mathbf{R}^{2}$, Sivakumar. $\mathbf{N}^{3}$, Manoj Prabhakar B.S ${ }^{4}$, Pradab.R ${ }^{5}$ \\ 1, 2, 3,4,5 Assistant Professor, Mechanical Engineering, CSI College of Engineering, Ketti, The Nilgiris, Tamilnadu.
}

\begin{abstract}
In this paper our work was to study the application and the uses of unmanned aerial vechicle insecticide spraying. Indian agriculture needed production and protection materials to achieve high productivity. Indian farmers are not rich to buy costly equipment for spraying pesticides or insecticides in their farm. Use of pesticides plays important role in agricultural fields for a better crop yielding. A quadcopter, also called a quad rotor helicopter or quad rotor, is a multirotor helicopter that is lifted and propelled by four rotors. Quadcopters are classified as rotorcraft, as opposed to fixed-wing aircraft, because their lift is generated by a set of rotors (vertically oriented propellers). The paper proposes a new strategy to replace humans in various agricultural operations like detection of presence of pests, spraying of pesticides, spraying of fertilizers, etc.Quadcopter Drone is working principle on aviation, It means airborne, airplane, etc.This research focused on develops a remotely operated Quadcopter system. . The paper proposes a new strategy to replace humans in various agricultural operations like detection of presence of pests, spraying of pesticides, spraying of fertilizers, etc.The Quadcopter is controlled through Graphical User Interface (GUI). Communication between GUI and Quadcopter is done by using wireless communication system. The Quadcopter balancing condition is sensed by KK2 multi controller. For smooth landing, Quadcopter is equipped with ultrasonic sensor.All Signals from sensors are processed by KK2 Circuit board.The experiment shows that Quadcopter can hover with maintain it balancing and stability. Quadcopter can accept load disturbance up to $250 \mathrm{~g}$ during it Hover condition. Maximum operated time of Quadcopter is six minutes using 3200mAh Lipo battery and operate time can be increase by using largest battery Capacity. This project is to mainly overcome the ill-effects of pesticides on human beings.
\end{abstract}

Keywords: Unmanned Aerial Vehicle, Lipo battery, KK2 circuit board, GUI

\section{INTRODUCTION AND OVERVIEW:}

The UAV is commonly known as UAS or, more precisely, as a drone, which can fly autonomously. UAV traditionally has a steadfast backdrop in aerodynamics, momentum arrangements, and theoretical physics practical rules. In the recent few years, it has also been a band of smart agriculture. UAVs can correctly receive and equitably distribute this information, allowing producers to take steps based on individual soil circumstances. Precision farming, crop monitoring, geographical tracking, and field mapping are a few sectors where UAVs play a massive role. In image analysis, the photos are promptly captured by UAV camera, and it is then adjusted to enhance the quality using various software to explore typically and precisely the target area and correctly recognize the unconventional objects. Agricultural UAV properly utilizes image processing to save an accurate record of plants' growth, quality of the active area, pest control, etc.

More than 60 percent of the planet's population naturally depends on agriculture for survival in the current world. The modernization of agriculture is mandatory because it increases production and food supply. Spraying pesticides and fertilizer remains quite an uncomplicated task for UAVs. UAVs for spraying help restrict human touch with fertilizers, pesticides, and other dangerous substances. Some developing countries like India, China, South Korea, Brazil, Sri Lanka, and Japan have already commenced agricultural monitoring by UAVs substantially. A diagram of the agricultural control system is shown precisely in Figure 1. 
DOI: 10.17148/IARJSET.2021.8936

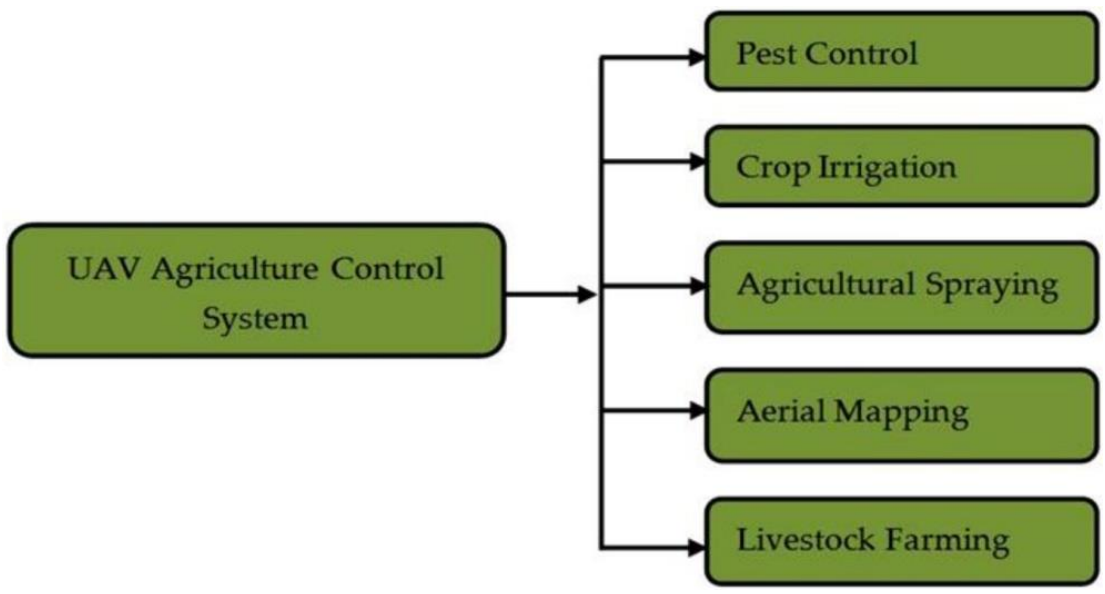

Figure 1. Diagram of the agricultural control system

The application of the pesticides is very important for the better crop yields. The manual spraying of pesticides using hand pump is quite difficult and dangerous to health because of directly contact with those chemical contain. Also manual spraying causes the non-uniformly spraying, excess spraying and this may cause to reduce the crop yields. In this paper, we describe an architecture based on unmanned aerial vehicles (UAVs) which can be employed for agricultural applications where UAVs are used for spraying pesticides on crops. The process of spraying is done by the drone by the controlling it by the radio controller using the wireless communication by means of the transmitter and the receiver.

As UAVs are remotely guided, they genuinely need a data link with the ground control system, and the direct connection can be compromised by unwanted users. The essence of data is vital and must be the focus of UAV use judgments. However, the cons will not be the prime focus of this paper. If UAV technology continues to grow, the expenses and limitations of agriculture will definitely decrease.

This paper typically portrays the character of suitable UAVs for a specific agricul- tural purpose. Furthermore, it will be explicit about assuming which archetype of a UAV is imperative for particular farming works.

One is single-dual rotors (Figure 2a), and the other is multi-rotor. The most reliable wing-based UAV is the multi-rotor UAV. Multi-rotors are quick and agile platforms and can satisfactorily perform demanding maneuvers. It is the most significant technological rise in the last decade. There are equally numerous models of Multi-rotor UAVs i.e., Tricopter (Figure 2b), Quadcopter (Figure 2c), Hexacopter (Figure 2d) and Octocopter (Figure 2e).

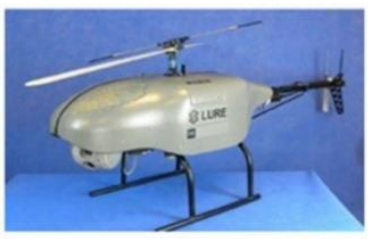

(a)

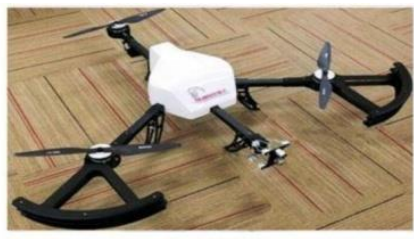

(b)

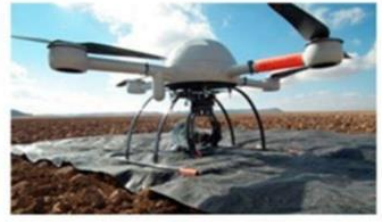

(c)

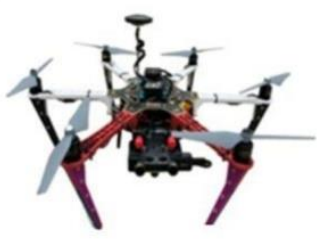

(d)

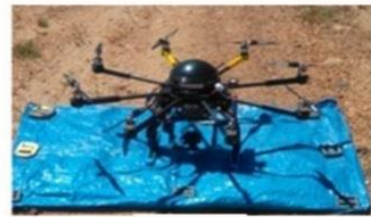

(e)

Figure 2. Types of rotary wings. Single-dual rotors [26] (a), Tricopter [30] (b), Quadcopter [28] (c), Hexacopter [29] (d), and Octocopter [9] (e). 


\section{DOI: 10.17148/IARJSET.2021.8936}

A quadcopter also called a quadrotor helicopter or quadrotor is a multirotor helicopter that is lifted and propelled by four rotors. Quadcopters are classified as rotorcraft, as opposed to fixed-wing aircraft, because their lift is generated by a set of rotors.

Quadcopters generally use two pairs of identical fixed pitched propellers; two clockwise (CW) and two counterclockwise $(\mathrm{CCW})$. These use independent variation of the speed of each rotor to achieve control. By changing the

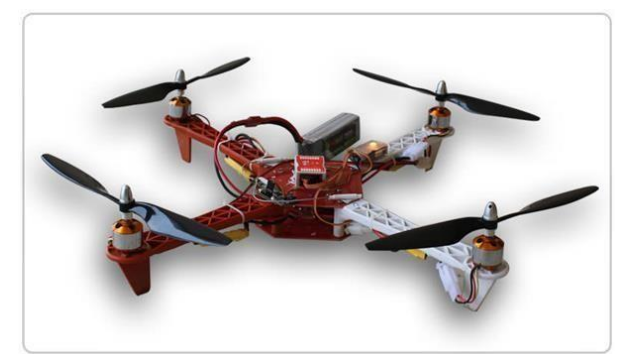

speed of each rotor it is possible to specifically generate a desired total thrust; to locate for the centre of thrust both laterally and longitudinally; and to create a desired total torque, or turning force.

A number of manned designs appeared in the 1920s and 1930s. These vehicles were among the first successful heavierthan-air vertical take-off and landing (VTOL) vehicles. However, early prototypes suffered from poor performance, and latter prototypes required too much pilot work load, due to poor stability augmentation and limited control authority.

At a small size, quadcopters are cheaper and more durable than conventional helicopters due to their mechanical simplicity. Their smaller blades are also advantageous because they possess less kinetic energy, reducing their ability to cause damage. For small-scale quadcopters, this makes the vehicles safer for close interaction. It is also possible to fit quadcopters with guards that enclose the rotors, further reducing the potential for damage. However, as size increases, fixed propeller quadcopters develop disadvantages over conventional helicopters. Increasing blade size increases their momentum. This means that changes in blade speed take longer, which negatively impacts control. At the same time, increasing blade size improves efficiency as it takes less energy to generate thrust by moving a large mass of air at as low speed than by moving a small mass of air at high speed. Therefore, increasing efficiency comes at the cost of control. Helicopters do not experience this problem as increasing the size of the rotor disk does not significantly impact the ability to control blade pitch.

Due to their ease of construction and control, quadcopter aircraft are frequently used as amateur model aircraft projects. In a Quadcopter, every rotor plays a vital role in the desired direction and proper balance of vehicles and lifting payloads. A Quadcopter genuinely has two unique designs; (i) plus model (Figure 3a), and (ii) cross model (Figure 3b). The Quadcopter with cross configuration is more stable than the plus configuration Quadcopter, for the equitable distribution of rotor force during hover. Two counter-rotating propellers are there for each particular side's pitch and roll axis for the cross configuration. However, plus configuration has only one on each side. Pitching ahead in this practical form establishes minor stability in the roll axis. In short, the four rotors sufficiently advance a Quadcopter to a very well-built flying machine. A pair of the rotors turns clockwise $(\mathrm{CW})$, and the other pair rotates counterclockwise $(\mathrm{CCW})$. Furthermore, that progressively eliminates the proportional torque naturally generated by the rotating-rotors. It also assists in excluding the boom requirement, which properly accommodates the Quadcopter, enhancing stability.

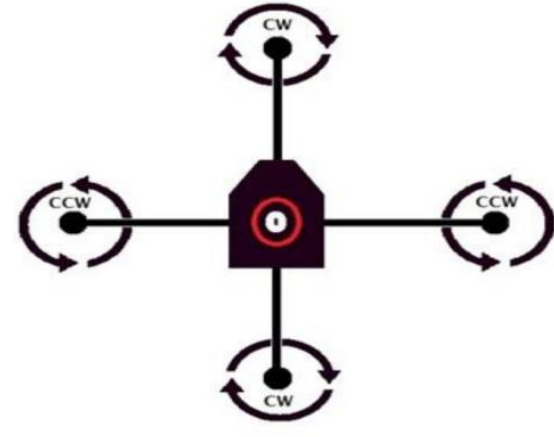

(a)

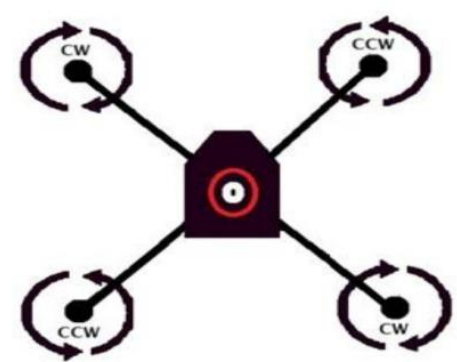

(b)

Figure 3. Quadcopter Flight Configurations. Plus configuration (a) and Cross configuration (b) (photo illustrated by the authors). 


\section{International Advanced Research Journal in Science, Engineering and Technology}

Vol. 8, Issue 9, September 2021

DOI: 10.17148/IARJSET.2021.8936

\section{Frame:}

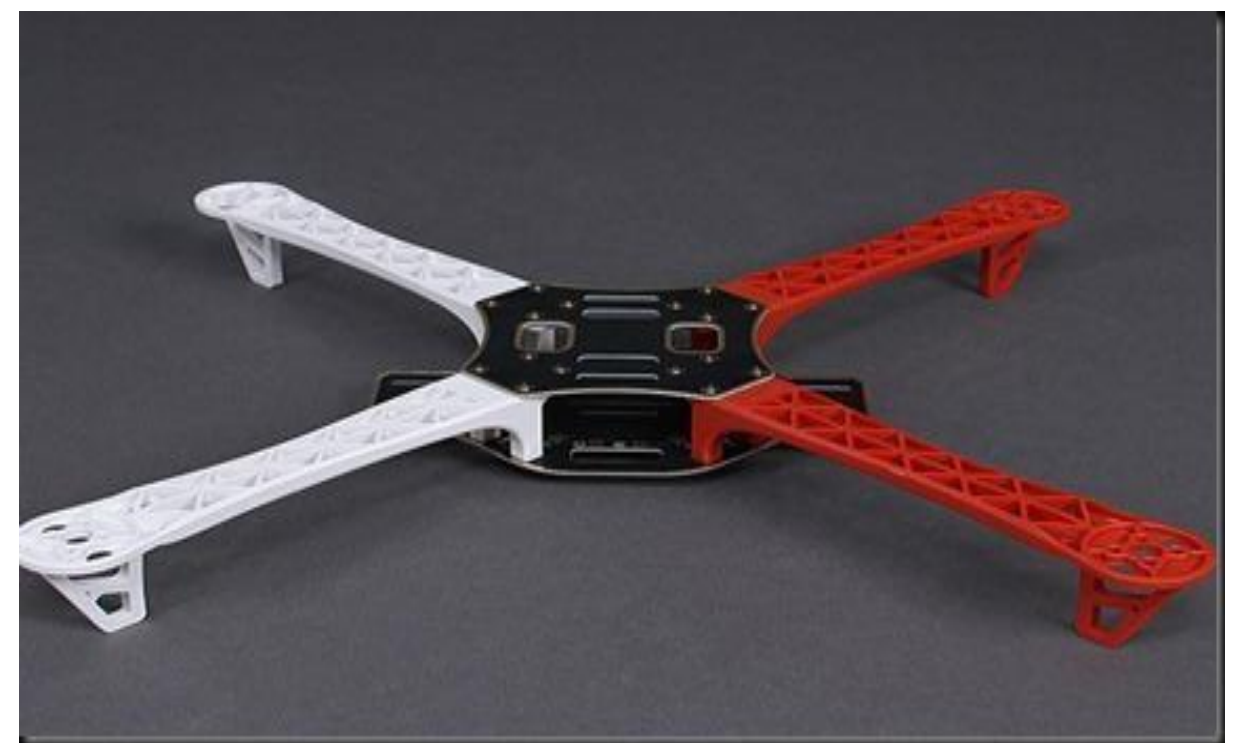

Figure 4. Frame of quadcopter

This is the most important basic part of a Quadcopter. As the name indicates, the copter has 4arms. The frame should be light as well as rigid to host a LIPO battery, 4 BLDC motors, 4 ESC \&controller.

You can build your own frame using Aluminium or wood channels. But I suggest you to go for a readymade one like F450 FRAME which is easy to assemble.

The frame arms are made of ultra strength material to survive any crash. The frame boards are high strength compound PCB frames, which makes wiring of ESCs and battery safer and easier. To make your flight colourful the frame arms come with different colours.

\section{KK2.1.5 Circuit Board:}

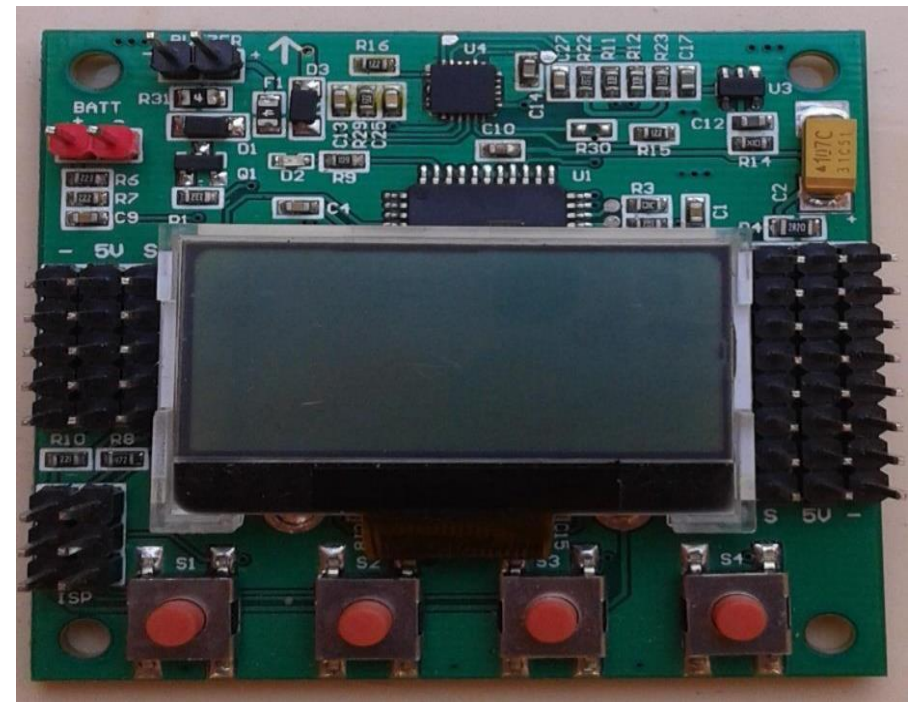

Figure 5. KK2.1.5 Circuit Board

The KK.2.1.5 Multi-Rotor controller manages the flight of (mostly) multi-rotor Aircraft (Tricopters, Quadcopters, Hex copters etc). Its purpose is to stabilize the aircraft during flight and to do this, it takes signals from on-board gyroscopes (roll, pitch and yaw) and passes these signals to the Atmega324PA processor, which in-turn processes signals according 


\title{
International Advanced Research Journal in Science, Engineering and Technology
}

\author{
Vol. 8, Issue 9, September 2021
}

DOI: 10.17148/IARJSET.2021.8936

the users selected firmware(e.g.Quadcopter) and passes the control signals to the installed Electronic Speed Controllers (ESCs) and the combination of these signals instructs the ESCs to make fine adjustments to the motors rotational speeds which 15 in-turn stabilizes the craft.

The KK2.1.5Multi-Rotor control board also uses signals from your radio system via a receiver (Rx) and passes these signals together with stabilization signals to the Atmega324PA IC via the aileron; elevator; throttle and rudder user demand inputs. Once processed, this information is sent to the ESCs which in turn adjust the rotational speed of each motor to control flight orientation (up, down, backwards, forwards, left, right, yaw)

The most common Control board widely used is KK control board. Many versions are available.

The models available are KK2.0, KK2.1.5 \& KK2.1HC.

The first 2 models have on board LCD this may break when the copter crashes (for a beginner). To avoid this the third model was introduced where the LCD unit is remotely connected.

The KK board has built in Gyros, accelerometers \& a Microcontroller to control the ESCs.

The LCD screen and built in software makes install and setup easier than ever. A host of multi-rotor craft types are pre-installed.

Simply select your craft type, check motor layout/propeller direction, calibrate your ESCs and radio and you're ready to go! All of which is done with easy to follow on screen prompts!

The original KK gyro system has been updated to an incredibly sensitive dual chip 3 Axis gyro and single chip 3 axis accelerometer system making this the most stable KK board ever and allowing for the addition of an Auto- level function.

At the heart of the KK2.0 is an Atmel Mega324PA 8-bit AVR RISC-based microcontroller with 32k of memory.

An additional 2 motor output channels have been added to the KK2.0 allowing for a total of 8 motors to16 be controlled.

A handy Pezos buzzer is also included with the board for audio warning when activating and deactivating the board.

If you're new to multi-rotor flight or have been unsure about how to setup a KK board then the KK2.0 was built for you.

The 6 Pin US asp AVR Programming interface ensures future software updates will be quick and easy.

The version KK2.1.5 has upgraded gyro system (6050 Microprocessor system) making this the most stable KK board ever and allowing for the addition of an auto-level function.

At the heart of the KK2.1.5 is an Atmel Mega 644PA 8-bit AVR RISC-based microcontroller with 64k of memory. An additional polarity protected header has been added for voltage detection, so no need for on- board soldering.

The KK2.1.5addedpolarityprotectionto the voltage sense header and a fuse protected buzzer outputs, in case something is accidentally plugged in incorrectly.

\section{Brushless Motor:}

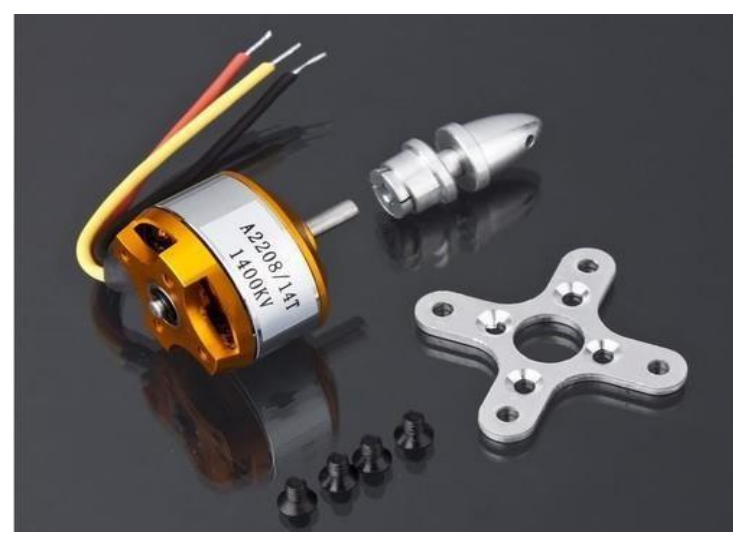

Figure 6. Brushless Motor

A Brushless DC motor is an internally commutated electric motor designed to be run from a direct current power source. Brushed DC motors can be varied in speed by changing the operating voltage or the strength of the magnetic field. Depending on the connections of the field to the power supply, the speed and torque characteristics of a brushed motor can be altered to provide steady speed or speed inversely proportional to the mechanical load. Since the brushes wear down and require placement, brushless DC motors using power electronic devices have displaced brushed motors from many applications. 


\section{International Advanced Research Journal in Science, Engineering and Technology}

Vol. 8, Issue 9, September 2021

DOI: $10.17148 /$ IARJSET.2021.8936

A problem with the motor shown above is that when the plane of the coil is parallel to the magnetic field-i.e. when the rotor poles are 90 degrees from the stator poles - the torque is zero. In the pictures above, this occurs when the core of the coil is horizontal - the position it is just about to reach in the last picture on the right. The motor would not be able to start in this position. However, once it was started, it would continue to rotate through this position by momentum. These field effects are reversed when the direction of spin is reversed. It is therefore difficult to build an efficient reversible commutated dynamo, since for highest field strength it is necessary to move the brushes to the opposite side of the normal neutral plane.

The effect can be considered to be somewhat similar to timing advance in an internal combustion engine. Generally a dynamo that has been designed to run at a certain fixed speed will have its brushes permanently fixed to align the field for highest efficiency at that speed.

\section{Speed control:}

An electronic speed control or ESC is an electronic circuit with the purpose to vary an electric motor's speed, its direction and possibly also to act as a dynamic brake. ESCs are often used on electrically powered radio controlled models, with the variety most often used for brushless motors essentially providing an electronically generated threephase electric power low voltage source of energy for the motor.

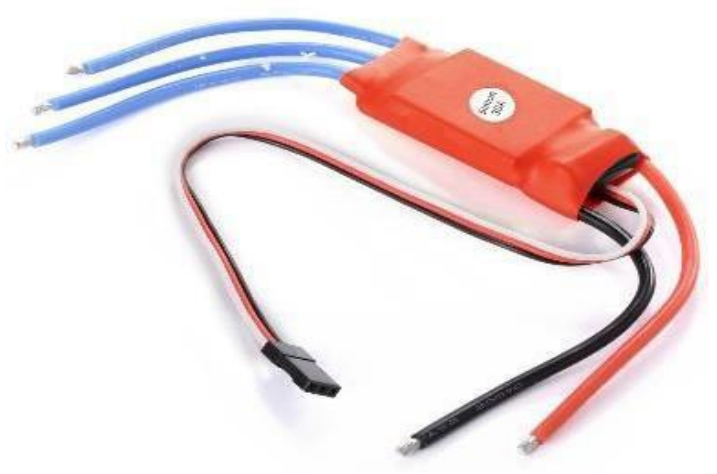

Figure 7. Speed control

\section{Battery:}

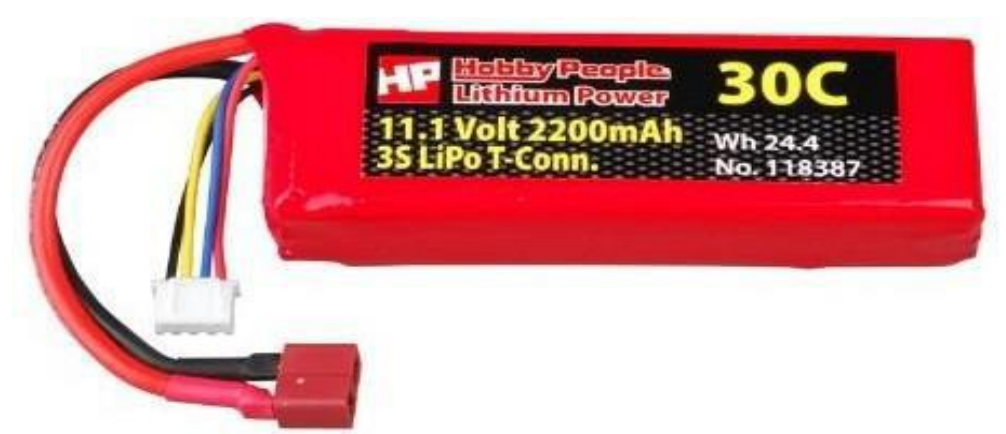

Figure 8. LiPo Battery

Lithium Polymer-LIPO is a type of rechargeable battery that has taken the electric RC world by storm, especially for Quadcopters. They are the main reason electric flight is now a very viable option over fuel powered models.

LiPo batteries are light in weight $\&$ hold huge power in a small package. They have high discharge rates to meet the need of powering quadcopters.

Remember LiPo batteries are much expensive \& have life time of only 300 to 400 charge cycles.

Special care to be taken to Charge, discharge or store the LiPo. Because of the volatile electrolyte used in LiPo they can burst or catch fire easily when mishandled 


\section{International Advanced Research Journal in Science, Engineering and Technology}

Vol. 8, Issue 9, September 2021

\section{DOI: $10.17148 / I A R J S E T .2021 .8936$}

Unlike conventional NiCad battery cells that have a voltage of 1.2 volts per cell, LiPo battery cells are rating those 3.7 volts per cell. So you get in multiples of $3.7 \mathrm{v}$ like $7.4 \& 11.1 \mathrm{v}$ batteries.

RC LiPo battery packs will have at least two or more cells hooked up in series to provide higher voltages. So an $11.1 \mathrm{v}$ battery, which is widely used, has 3 cells x 3.7 volts (3S)

Capacity indicates how much power the battery pack can hold and is indicated in milliamp hours (mAh).

A $3200 \mathrm{mAh}$ would be completely discharged in one hour with a 3200 milliamp load placed on it. If this same battery had an 1100 milli amp load placed on it, it would take 2 hours to drain down.

If you want to increase your flight time use more capacity battery like $3000 \mathrm{maH}$.

LiPo batteries can be found in packs of everything from a single cell (3.7V) to over 10 cells $(37 \mathrm{~V})$. The cells are usually connected in series, making the voltage higher but giving the same amount of amp-hours.

Discharge rate is simply how fast a battery can be discharged safely. In the RC LiPo battery world it is called the "C" rating. Remember you should never discharge a LiPo BATTERY BELOW 80\% OF ITSCAPACITY.

\section{Remote Control:}

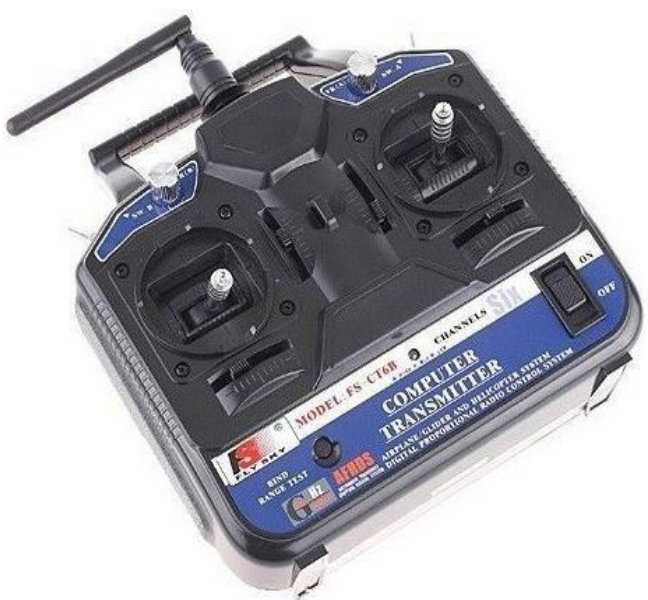

Figure 9. Remote Control

You need a RC Transmitter $(2.4 \mathrm{GHz} \mathrm{RC}$ radio transmitter) to direct the quadcopter direction and position. A $2.4 \mathrm{GHz}$ $\mathrm{RC}$ radio receiver on the quadcopter receives commands from the RC transmitter on the ground. (One way link). You get a Receiver along with the corresponding Transmitter.

The transmitter is the hand-held controller you use to remotely control your craft. The transmitters have two sticks, two trim buttons or a slider per stick, a number of switches, a display, and a power button.

Transmitters and receivers need a frequency range to operate and the new frequency range is $2.4 \mathrm{GHz}$, with digital spectrum modulation.2.4GHZ is the ISM (Industrial, Scientific \& Medical) Radio band which needs no license to operate.

\section{Receiver:}

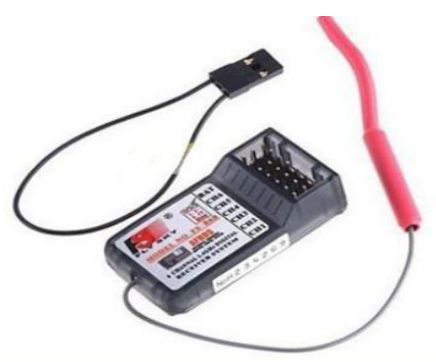

Figure 10. Receiver

Receiver type and multi-rotor type are very easily selected via the small DIP switches and calibration is also extremely easy with the set button. Once calibrated sensitivity is also a very simple affair with just one small adjuster on the front of the unit making this flight controller one of the easiest to set up ever. 


\section{International Advanced Research Journal in Science, Engineering and Technology}

Vol. 8, Issue 9, September 2021

DOI: 10.17148/IARJSET.2021.8936

\section{Layout of Quadcopter:}

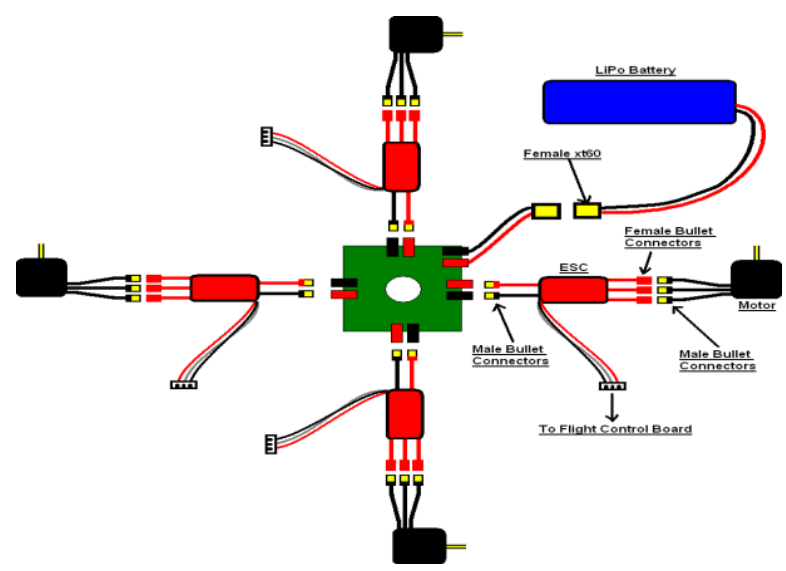

Figure 11.Layout of the Quadcopter

\section{APPLICATIONS:}

\section{Research platform:}

Quadcopters are a useful tool for university research to test and evaluate wide as in a number of different fields, including flight control theory, navigation, real time systems, and robotics. In recent years many universities have shown quadcopters performing increasingly complex aerial many curves.

\section{Military and law enforcement:}

During the Battle of Mosul it was reported that commercially available quadcopters and drones were being used by Islamic State of Iraq and the Levant (ISIL) as surveillance and weapons delivery platforms using improvised cradles to drop grenades and other explosives. The ISIL drone facility became a target of Royal Air Force strike aircraft.

\section{Photography:}

2016 model DJI Phantom 4 quadcopter with a high definition 4k stabilized video and still camera, GPS stabilization and automatic obstacle avoidance.

The largest use of quadcopters in the USA has been in the field of aerial imagery. Quadcopter UAVs are suitable for this job because of their autonomous nature and huge cost savings. Drones have also been used for light-painting photography

\section{CONCLUSION:}

In this paper we have studied of a drone mounted spraying mechanism for Agricultural purpose and for spraying disinfectants and application of qudacopter drone. This method of spraying pesticides on Agricultural fields reduces the number of labours, time, cost and the risk involved to the personnel involved in spraying the liquids. This drone can also be used in spraying disinfectant liquids over buildings, water bodies and highly populated areas.

\section{REFERENCES:}

1. Kim, J.; Kim, S.; Ju, C.; Son, H.I. Unmanned Aerial Vehicles in Agriculture: A Review of Perspective of Platform, Control, and Applications. IEEE Access 2019, 7, 105100-105115. [CrossRef]

2. Spoorthi, S.B.; Shadaksharappa, S.S.; Manasa, V.K. Freyr drone: Pesticide/fertilizers spraying drone-An agricultural approach. In Proceedings of the 2nd International Conference on Computing and Communications Technologies (ICCCT), Chennai, India, 23-24 February 2017; pp. 252-255.

3. Stojcsics, D.; Domozi, Z.; Molnár, A. Automated evaluation of agricultural damage using UAV survey. Acta Univ. Sapientiae Agric. Environ. 2018, 10, 20-30. [CrossRef]

4. Yanliang, Z.; Qi, L.; Wei, Z. Design and test of a six-rotor unmanned aerial vehicle (UAV) electrostatic spraying system for cropprotection. Int. J. Agric. Biol. Eng. 2017, 10, 68-76. [CrossRef]

5. Marinello, F.; Andrea, P.; Alessandro, C.; Luigi, S. Technical analysis of unmanned aerial vehicles (drones) for agricultural applications. Eng. Rural Dev. 2016, 15, 870-875.

6. Rahman, M.F.F.; Fan, S.; Zhang, Y.; Chen, L. A Comparative Study on Application of Unmanned Aerial Vehicle Systems in Agriculture. Agriculture 2021, 11, 22. https://doi. org/10.3390/agriculture11010022

7. Hari Krishna J; Pramoth R; Anoint Joshua Paul J; Abhishek S; Prabhu T. "Soldiers health monitoring and tracking system using IOT and UAV". International Research Journal on Advanced Science Hub, 2, Special Issue ICSTM 12S, 2020, 78-81. doi: 10.47392/irjash.2020.265. 